\title{
Exploring Fingers' Limitation of Texture Density Perception on Ultrasonic Haptic Displays
}

\author{
Farzan Kalantari ${ }^{1}$, David Gueorguiev ${ }^{2}$, Edward Lank $^{3}$, Nicolas Bremard ${ }^{1}$, and \\ Laurent Grisoni ${ }^{1}$ \\ 1 University of Lille - Science \& Technology, CNRS, Lille, France \\ farzan.kalantari@ed.univ-lille1.fr, nicolas.bremard@inria.fr, \\ laurent.grisoni@univ-lille1.fr \\ 2 Max-Planck Institute for Intelligent Systems, Stuttgart, Germany \\ dgueorguiev@is.mpg.de \\ 3 University of Waterloo, Waterloo, Canada \\ lank@uwaterloo.ca
}

\begin{abstract}
Recent research in haptic feedback is motivated by the crucial role that tactile perception plays in everyday touch interactions. In this paper, we describe psychophysical experiments to investigate the perceptual threshold of individual fingers on both the right and left hand of right-handed participants using active dynamic touch for spatial period discrimination of both sinusoidal and square-wave gratings on ultrasonic haptic touchscreens. Both one-finger and multi-finger touch were studied and compared. Our results indicate that users' finger identity (index finger, middle finger, etc.) significantly affect the perception of both gratings in the case of one-finger exploration. We show that index finger and thumb are the most sensitive in all conditions whereas little finger followed by ring are the least sensitive for haptic perception. For multi-finger exploration, the right hand was found to be more sensitive than the left hand for both gratings. Our findings also demonstrate similar perception sensitivity between multi-finger exploration and the index finger of users' right hands (i.e. dominant hand in our study), while significant difference was found between single and multi-finger perception sensitivity for the left hand.
\end{abstract}

Keywords: Haptic display; Tactile perception; Ultrasonic vibration; Finger sensitivity; Spatial texture density

\section{Introduction}

Current commercial touchscreen devices rarely provide a compelling haptic feedback to human fingers despite the use of touch as input; haptic feedback is typically limited to vibration. As Buxton et al. [5] investigated in 1985, flat touchscreens need haptic feedback in order to ease end users' common interaction tasks, to enhance the efficiency of interfaces, and to increase the realism of visual environments. Therefore, researchers have explored different technologies to generate dynamic haptic feedback to enhance input on touchscreen devices. 
Within this space of dynamic haptic effects, different technologies are commonly used. First, vibrotactile actuators such as solenoids, vibrotactile coils, and ERM motors can be utilized for tactile rendering on touchscreens as discussed in [6]. These actuators are used presently on smartwatches, mobile phones and tablets, but typically provide for on-or-off sensation. Alongside vibrotactile actuation, two techniques, electrostatic-vibration $[2,18]$ and electroadhesion [22] use electrostatic force generated, respectively, by applying a voltage to the screen surface or by applying DC excitation of the tactile display. Both of these techniques increase the friction between the finger and the interaction surface when activated, thus varying the perceived stickiness of the surface. Finally, a fourth type of haptic feedback leverages ultrasonic vibrations to create an air-gap between a user's finger and the display to reduce friction when activated, a phenomenon called the "squeeze film effect" $[4,1,7,28]$. In the remainder of this paper, we are particularly interested in the user's tactile perception of the latter technology.

It is well-documented in literature that the human sense of touch has a fundamental role in the haptic perception of different surfaces. Touch is quite sensitive in perceiving different materials [10] and textures [16], and we leverage this sensitivity in haptic effects by taking into account its fundamental limits [23]. The texture perception of the human sense of touch remains a complex phenomenon which varies between different people and is mediated by the user's fingers' mechanoreceptors [21].

This complexity of touch perception has resulted in various investigations to better understand and explore haptic perception difficulties, particularly on tactile surfaces. Yoshioka et al. [29] have shown that the neural mechanisms underlying texture perception of a variety of real textured surfaces and objects differ between direct touch (through a finger) and indirect touch (through a probe). Hughes et al. [12] investigated participants' abilities to discriminate spatial density gradients of different textures. Nefs et al. [20] measured discrimination thresholds for sinusoidal gratings using active dynamic touch and found that amplitude differences as small as $2 \mu \mathrm{m}$ can be detected with spatial periods between 0.25 and $1 \mathrm{~cm}$. Verrillo et al. [25] studied the relationship between vibration frequency and perceived intensity of the stimuli and showed that it obeys a power law function with an exponent of 0.89 for frequencies under $350 \mathrm{~Hz}$. Wijekoon et al. [27] demonstrated that there are significant correlations between intensity perception and signal frequency and amplitude of texture waveform for texture perception on electrovibration haptic displays, and the highest sensitivity was found at a frequency of $80 \mathrm{~Hz}$.

In the case of tactile perception of ultrasonic haptic displays, those that leverage the squeeze film effect, several studies have also been performed on touch perception. Biet al. [3] studied the differential sensory thresholds for the spatial periods of real and virtual square-wave gratings on an ultrasonic haptic plate. Kalantari et al. [14] studied the limitation of tactile elements for texture perception and how to optimize interaction performance of end users through the perception of different haptic effects [15], as well as how tactile and auditory signals can be combined to enhance the user's spatial perception in musical 
interactions on ultrasonic displays [13]. Gueorguiev et al. [9,11] investigated the tactile perception of transient changes of different frictional signals on ultrasonic based haptic devices.

Despite all of this work, however, in all of the mentioned studies only one finger (index in most cases) for texture perception of tactile surfaces has been examined; we are aware of no work that has contrasted finger sensitivity, nor any work that explores single versus multi-finger sensitivity. Given that single-touch interaction need not be limited to the index finger, and given the prevalence of multi-touch as an input paradigm on touch screens, one can ask the followings: Do we have identical texture perception among all our fingers and hands while interacting with a haptic display? Do we have the same sensory threshold for perceiving different kind of textures? What are the differences between the tactile perception of one-finger and multi-finger explorations on haptic displays? In this paper, we explore the limitation of individual human fingers and different hands on texture density perception in the case of two waveform types for ultrasonicbased haptic displays.

\section{Experiment}

We carried out a psychophysical experiment to explore the limitations of touch perception of different finger types (index, middle, etc.) in dynamic active touch. We investigated both single and multi-finger tactile explorations of sinusoidal and square-wave textures on ultrasonic-based tactile displays. In this study, texture is defined as the sequence of periodic haptic feedback effects generated by a specific type of signal waveform (such as square or sine) and accordingly its specific value of spatial period and amplitude. We have investigated the spatial period of determined textures (with a constant amplitude of $1.25 \mu \mathrm{m}$ peak to peak) which can be accurately perceivable by participants.

The experiment conformed to the principles of the Declaration of Helsinki and a general explanation of the experimental task was given to each participant before beginning the experimental procedure.

\subsection{Participants}

Fifteen healthy volunteers ( 5 females) from the age of 22 to 34 with a mean age of $28.4(\mathrm{SD}=3.48)$ took part in our experiment. By design, all of the participants were right-handed. The total experiment time was 50-60 minutes for each participant. Participants wore active noise-cancelling headphones (Panasonic RPDJS200, Japan) during the experiment, while Gaussian white noise was played at a comfortable listening level in order to prevent potential interference from external auditory cues.

\section{$2.2 \quad$ Experimental set-up}

We used an enhanced visual-tactile actuator $(E-V i T a)$, a tactile feedback display based on ultrasonic vibrations for haptic rendering [26]. E-ViTa is developed 
on a Banana $\mathrm{Pi}$, a single-board computer (Shenzhen LeMaker Technology Co. Ltd, China) with a $1 \mathrm{GHz}$ ARM Cortex-A7, dual-core CPU and 1 GB of RAM working in parallel with STM32f4 microcontroller (STMicroelectronics, France). The communication between the microcontroller and the single board computer is provided via the Serial Peripheral Interface (SPI) bus at $10 \mathrm{kHz}$. This singleboard computer is connected to a $12.5 \mathrm{~cm}$ capacitive touchscreen (Banana-LCD 5 "-TS, MAREL, China) for detecting the user's finger position on the display with a sampling frequency of $62 \mathrm{~Hz}$.

Ten $14 \times 6 \times 0.5 \mathrm{~mm}$ piezoelectric cells actuate a $154 \times 81 \times 1.6 \mathrm{~mm}$ fixed glass plate, resonating at $60750 \mathrm{~Hz}$ with a half wavelength of $8 \mathrm{~mm}$. A power electronic circuit converts a $12 \mathrm{~V}$ DC voltage source into an AC voltage, controlled in amplitude and frequency and supplied to the piezoelectric cells. The microcontroller synthesizes a pulse-width modulation (PWM) signal to drive a voltage inverter that actuates the piezoceramics. The detailed structure of E-ViTa haptic display is illustrated in figure 1 .
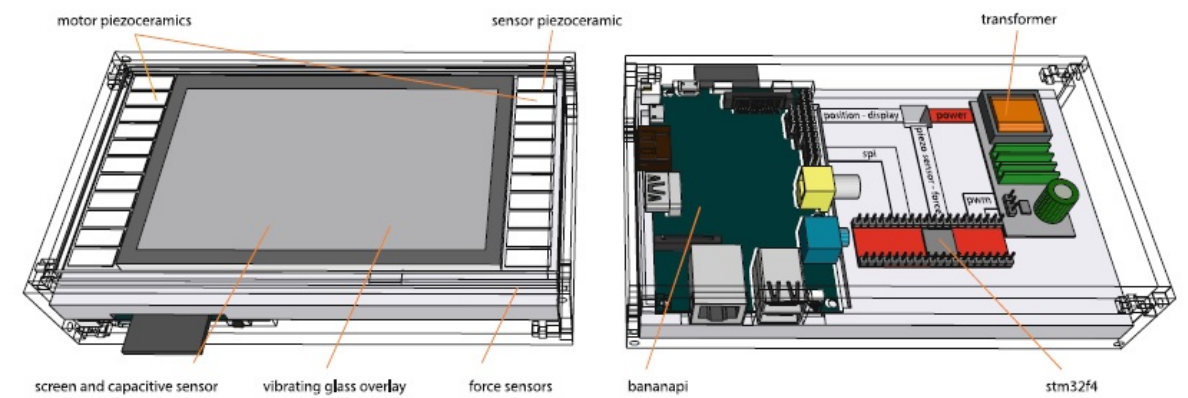

Fig. 1: Structure of the E-Vita ultrasonic based haptic display used in our experiment [26]

\subsection{General procedure}

A one-up-one-down staircase procedure (adaptive procedure) with fixed step sizes, commonly used in psychophysics $[24,17]$ was used in our investigation. In this procedure the stimulus level at any trial is determined by the previous response of a participant. The 1-up-1-down staircase procedure offers the compelling advantage of reducing the total time of our experiment, since we investigate a high number of trials and conditions for each participant.

The stimuli consisted of textures with sinusoidal and square wave gratings, which were tested on all fingers of both hands. Tactile exploration was also performed with the right and left hands (multi-finger exploration) for the two types of gratings. In the latter experimental situation, the participants were asked to use all their fingers except thumb in order to have sufficient active region of haptic feedback on the E-ViTa 5" display. The procedure for each 
finger continued until one of the following predefined conditions was obtained: maximum number of 30 trials or five consecutive turnover points (reversals); i.e. when a participant's response was different from the preceding trial (Fig. 2). Therefore, the total number of performed trials was equal to or less than 720 ( [10 finger types +2 hands $] \times 2$ signal waveforms $\times 30$ tries $=720$ ) for each participant in this experiment.

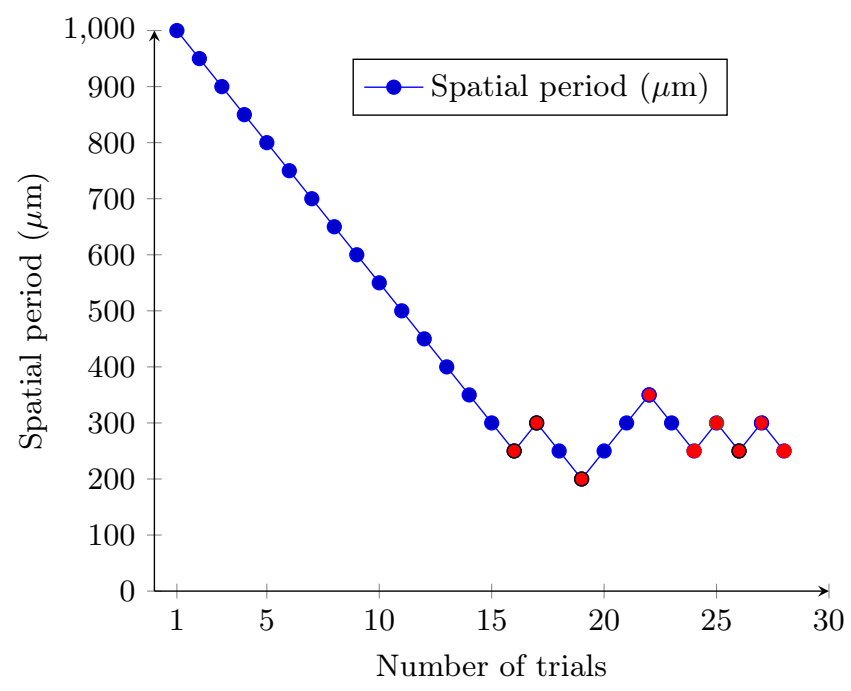

Fig. 2: Sample of data collected from a single participant using 1-up 1-down staircase procedure. Turnover points (reversals) are marked with red color. The sensory threshold was calculated by averaging the correct detected texture over the last five turnover points.

In our study, the initial texture (stimulus) had a spatial period of $1000 \mu \mathrm{m}$ for both sine and square grating and a constant amplitude of $1.25 \mu \mathrm{m}$ (peak to peak) with a response step of $50 \mu \mathrm{m}$ for each trial. This means that for each correct response the spatial period of our stimulus is decreased by $50 \mu \mathrm{m}$ and vise-versa for a wrong answer it will be increased by the same $50 \mu \mathrm{m}$ of stepsize. A reference texture for both sine and square gratings was set at a constant spatial period of $100 \mu \mathrm{m}$ and an amplitude of $1.25 \mu \mathrm{m}$ was also set. The E-ViTa ultrasonic haptic display was divided into 2 equal sections as illustrated in figure 3 . On the left half of the display, the reference texture was provided, while on the other half the stimulus which must be recognized and compared to the reference texture was placed. The participants were free to explore the surface as long as they wanted. At each trial they were asked to select the "identical" or "different" button regarding one-finger or multi-finger tactile perception of the provided (reference and stimulus) textures. The order of two gratings and users' fingers 
were randomized among participants during the trials in order to prevent any potential learning or habituation effects on the final results.

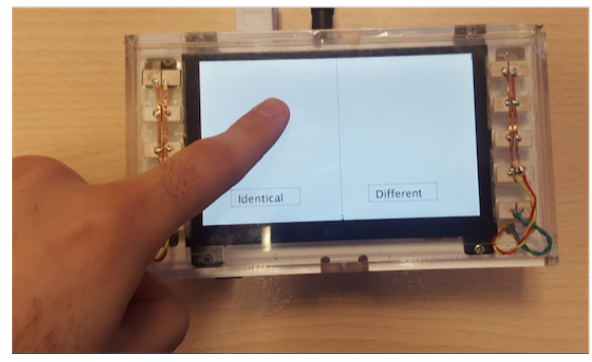

(a) One-finger exploration

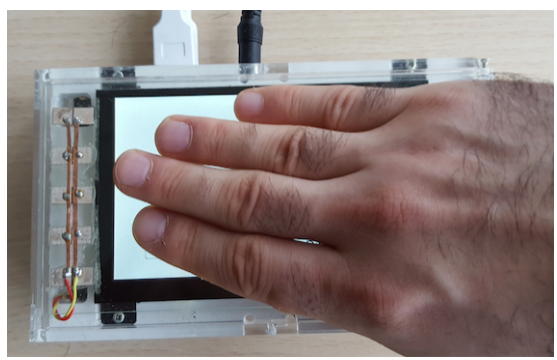

(b) Multi-finger exploration with four fingers (except thumb)

Fig. 3: The setup of our experiment in the two tactile exploration conditions

\section{Results}

The perceptual thresholds for both one-finger and multi-finger explorations of the spatial period of sinusoidal and square-wave gratings were analyzed.

\subsection{One-finger exploration}

The median values of the individual fingers' $50 \%$ perceptual thresholds for discriminating from the $100 \mu \mathrm{m}$ reference spatial period ranged between $150 \mu \mathrm{m}$ and $300 \mu \mathrm{m}$ for the sinusoidal grating and from $200 \mu \mathrm{m}$ to $350 \mu \mathrm{m}$ for the square grating. On both hands, we computed a Friedman non-parametric statistical test to estimate the effect of the finger type on the perceptual threshold (Fig. 4). The finger with which the exploration takes place was found to significantly affect the perception of the virtual gratings for both the sinusoidal $\left(\chi^{2}\right.$ $=50.35, \mathrm{p}<0.0001$ for the right hand and $\chi^{2}=36.72, \mathrm{p}<0.0001$ for the left hand) and square virtual grating $\left(\chi^{2}=34.10, \mathrm{p}<0.0001\right.$ for the right hand and $\chi^{2}=44.27, \mathrm{p}<0.0001$ for the left hand). The little finger was the least sensitive, i.e., had the highest perceptual threshold level in all conditions, and the ring finger was the second least sensitive in all conditions. For completeness, we also compared the sensory perception of the index finger, thumb and middle finger using a Wilcoxon matched-pairs signed rank test for all conditions (Fig. 5a). The pairwise comparison was performed across the two gratings and hands (60 pairs in total) in order to focus solely on the finger type. Index finger was found to be more sensitive than middle finger (Wilcoxon matched-pairs signed rank test: $\mathrm{N}=60, \mathrm{~W}=594, \mathrm{p}<0.0001)$ in all conditions. However, the comparison between the index finger and the thumb revealed no significant difference 
(Wilcoxon matched-pairs signed rank test: $\mathrm{N}=60, \mathrm{~W}=-293, \mathrm{p}=0.0797$ ) for the perceptual thresholds in any conditions. Similarly, the thumb and the middle finger differences in sensitivity did not rise to the level of statistical significance (Wilcoxon matched-pairs signed rank test: $\mathrm{N}=60, \mathrm{~W}=228, \mathrm{p}=0.0891$ ) considering all conditions. Altogether, our results indicate that the index finger and the thumb have similar sensitivity in all conditions and are therefore the most sensitive fingers for spatial period perception of both gratings among all fingers.
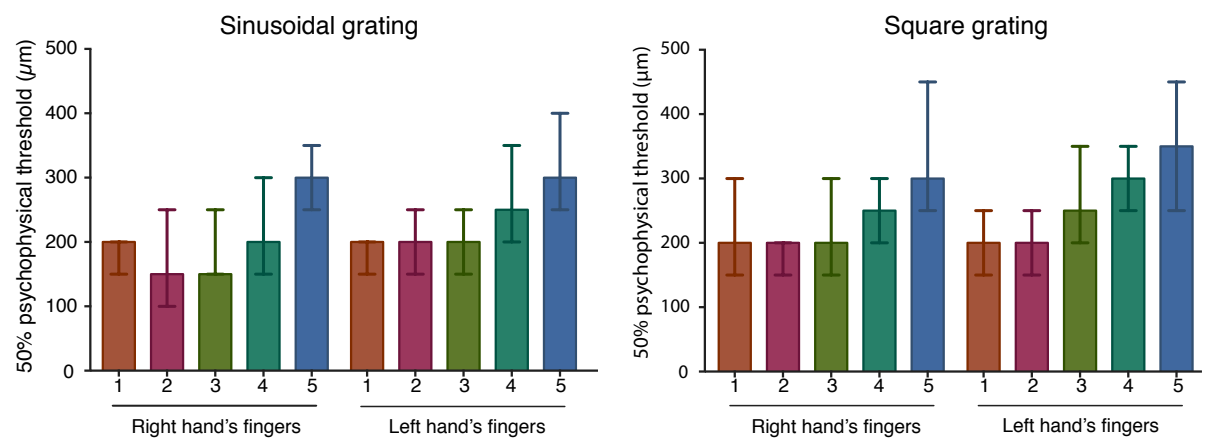

Fig. 4: The psychophysical threshold, computed as the $50 \%$ just noticeable difference between the comparison and reference stimuli of all finger types of both hands. The experiment was performed for sinusoidal and square virtual gratings. The finger types are as follow: (1) thumb, (2) index, (3)middle, (4) ring, (5) little. The boxplots show the median value and the error bars show the interquartile range.

To estimate the overall sensitivity thresholds of the right and left hands, we averaged the perceptual thresholds for all the fingers of each hand across the two types of gratings (Fig. 5b). We then compared them with a Wilcoxon matchedpairs signed rank test. The median spatial period that participants were able to discriminate from the $100 \mu \mathrm{m}$ reference stimulus $50 \%$ of the time $(50 \%$ just noticeable difference) was $230 \mu \mathrm{m}$ (IQR: 272.5-177.5) for the right hand and 255 $\mu \mathrm{m}$ (IQR: 310-200) for the left hand. The right hand of the participants, which was also their dominant hand, was significantly more sensitive than their left hand $(\mathrm{N}=30, \mathrm{~W}=383, \mathrm{p}<0.0001)$.

We used the same procedure to estimate the difference between the perception of square and sinusoidal gratings (Fig. 5c). In that case, the $50 \%$ perceptual threshold for discriminating from the reference grating was found to be $225 \mu \mathrm{m}$ (IQR: 267.5-177.5) for the sinusoidal grating and $245 \mu \mathrm{m}$ (IQR: 312.5-197.5) for the square grating. Participants were found to be significantly more sensitive to differences in the spatial period of the sinusoidal grating $(\mathrm{N}=30, \mathrm{~W}=366, \mathrm{p}<$ $0.0001)$. 

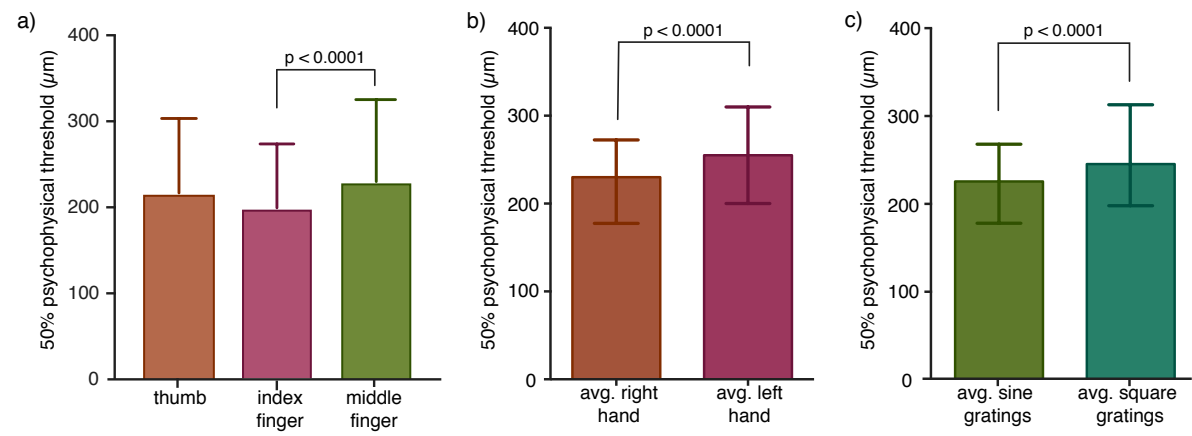

Fig. 5: a) The psychophysical threshold, computed as the $50 \%$ just noticeable difference for comparing the sensory thresholds (median \pm IQR) of thumb, index and middle fingers for both gratings. b) $50 \%$ psychophysical thresholds for all finger types across both types of gratings were averaged for each participants. The resulting thresholds (median \pm IQR) were compared between the right and left hand. c) The same procedure was performed to compare the psychophysical thresholds (median \pm IQR) between both types of gratings.

\subsection{Multi-finger exploration}

Participants performed the same task by exploring the actuated surface simultaneously with four fingers (except thumb). The results from multi-finger exploration showed similar trends to the exploration with one finger (Fig. 6). On the sinusoidal grating, the median $50 \%$ psychophysical threshold for discriminating between the $100 \mu \mathrm{m}$ grating was $150 \mu \mathrm{m}$ (IQR: 250-150) for the right hand and $250 \mu \mathrm{m}$ (IQR: 300-200) for the left hand. On the square grating, the median $50 \%$ psychophysical threshold was $200 \mu \mathrm{m}$ (IQR: 250-150) for the right hand and 250 $\mu \mathrm{m}$ (IQR: 300-200) for the left hand. As for the one finger exploration, significant differences were found between conditions. The right hand was found to be more sensitive on both types of gratings (Wilcoxon matched-pairs signed rank test: $\mathrm{N}=15, \mathrm{~W}=105, \mathrm{p}=0.0001$ for the sinusoidal grating and $\mathrm{N}=15, \mathrm{~W}=93, \mathrm{p}$ $=0.0015)$ for the square grating.

For both, the right and left hand, we compared the sensitivity of the index finger, which is the exploring finger in most studies on tactile perception and was also found to be the most sensitive in our experiments (Fig. 7). For the right hand, we did not observe a significant difference between the two exploratory techniques for any of the two types of gratings (Wilcoxon matched-pairs signed rank test: $\mathrm{N}=15, \mathrm{~W}=39, \mathrm{p}=0.09$ for the sinusoidal grating and $\mathrm{N}=15, \mathrm{~W}=17, \mathrm{p}$ $=0.30$ for the square grating). On the other hand, the left hand exhibited significant differences between the one-finger and multi-fingers explorations (Wilcoxon matched-pairs signed rank test: $\mathrm{N}=15, \mathrm{~W}=66, \mathrm{p}=0.001$ for the sinusoidal grating and $\mathrm{N}=15, \mathrm{~W}=48, \mathrm{p}=0.05$ for the square grating). Thus, multi-finger exploration was found to be statistically similar in sensitivity as the index finger when performed with the dominant hand while multi-finger significantly impaired par- 

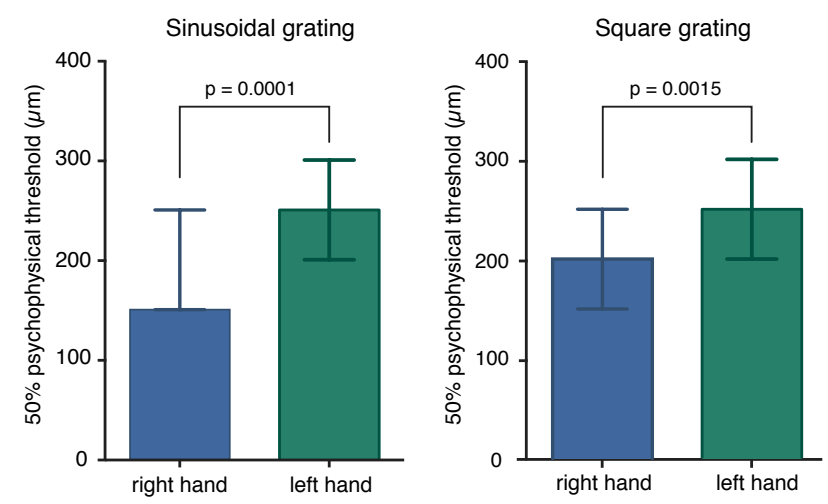

Fig. 6: Left: the $50 \%$ psychophysical thresholds (median \pm IQR) when exploration was simultaneously performed with four fingers on a sinusoidal grating were compared between the right and left hand. Right: The same comparison was made for the exploration of the square grating.

ticipant sensitivity to the spatial period of virtual gratings when performed with the non-dominant hand.
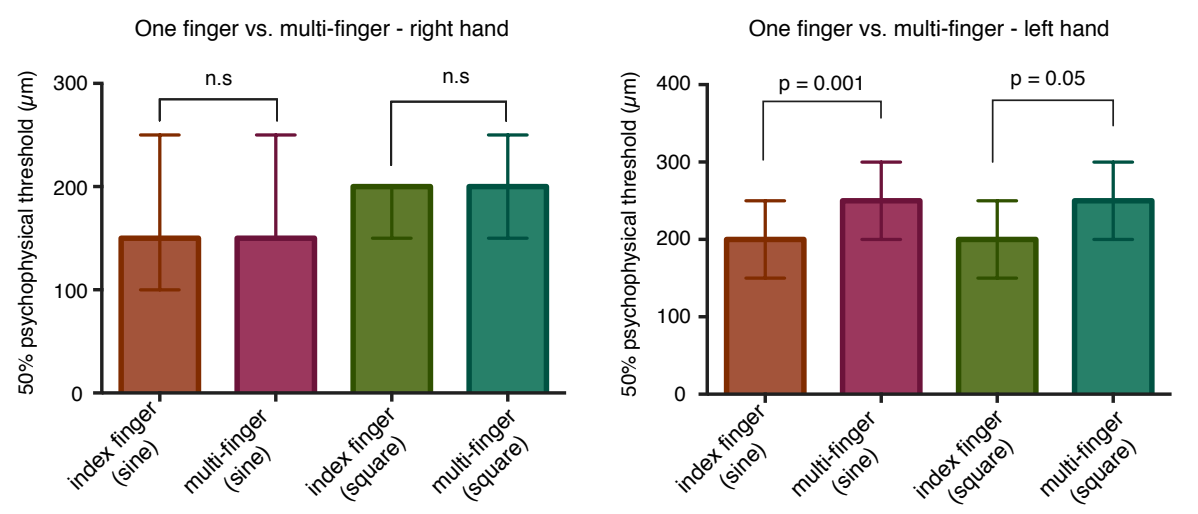

Fig. 7: Comparison of the $50 \%$ psychophysical thresholds (median \pm IQR) between when tactile exploration of the gratings was performed with the index finger and when it was performed with four fingers simultaneously. The comparison was made for both hands.

\section{Conclusion and perspective}

In this paper we investigated the effects and limitations of different human fingers for texture density perception for both single and multi-finger exploratory 
techniques and for both sine and square wave gratings of right-handed participants by leveraging ultrasonic vibration. To the best of our knowledge, this is the first study that systematically investigates different finger types' perception on haptic feedback touchscreens and the first study that explores multi-finger versus single-finger perception.

Our results indicate that the index and the thumb are the most sensitive fingers for perceiving differences in spatial textures for both sine and square virtual gratings; the little finger, followed by the ring, is the least sensitive for texture perception in one-finger exploration for the two types of gratings. The texture perception of the sinusoidal grating was also found to be more sensitive than the square grating. In multi-finger exploration, the dominant hand (right hand) was significantly more sensitive than the non-dominant hand for both gratings. This suggests that the dominant hand is more trained to perceive subtle spatial features. Furthermore, our findings showed that there was no significant difference between the sensitivity rate of the index finger (the most sensitive single-finger type) and multi-finger tactile exploration for users' dominant hand. In contrast, we observed significant differences between participants' left index finger and left-hand multifinger sensitivity perception. The dominant hand's preserved ability for spatial detection during multi-finger exploration may come from its ability to better perform synchronous dexterous motion. This enhanced perception sensitivity permits the dominant hand to control exploratory motion in order to optimize the consistency of tactile feedback across fingers. Given these results, we hypothesize the index finger has a major impact on the overall multi-finger sensitivity of the user's dominant hand for both types of gratings.

In the future, we would like to investigate if these results generalize to lefthanded users, which would confirm the importance of hand dominance. While hand dominance is most likely the driving force behind variable sensitivity, it is possible that perceptual differences could result from left-right physiological differences. A sufficiently large pool of left-handed participants is necessary to validate hand dominance versus left-right physiology. It would also be interesting to investigate in greater depth differences in the dynamics of multi-finger tactile exploration between both hands.

In terms of implications, for designers, these results provide guidance on the need to vary haptic stimuli depending on whether the stimulus is designed for the dominant or non-dominant hand, depending on whether it is designed for the index finger or for any finger, and depending on whether it is to be a single-finger of multi-finger interaction. As well, a potential use of different sensory thresholds of finger types, which we found in our study, is to leverage these sensitivities to novel finger identification techniques on tactile displays $[8,19]$ in order to allow users to perform different interaction tasks. For instance, possible gestures (such as: selecting an object, dragging or swapping) could be linked via textures to the fingers involved in the interaction.

Acknowledgments. The authors would like to thank the CNRS/IRCICA USR 3380 research center, which hosted the research. This work is partially 
funded by European ERDF grants (IRCICA, CPER MAUVE) and ANR funding agency. Researcher support was also provided by the Invited Researcher Program of Région Hauts-de-France.

\section{References}

1. Amberg, M., Giraud, F., Semail, B., Olivo, P., Casiez, G., Roussel, N.: Stimtac: A tactile input device with programmable friction. In: Proceedings of the 24th Annual ACM Symposium Adjunct on User Interface Software and Technology. pp. 7-8. UIST '11 Adjunct, ACM (2011)

2. Bau, O., Poupyrev, I., Israr, A., Harrison, C.: Teslatouch: Electrovibration for touch surfaces. In: Proceedings of the 23nd Annual ACM Symposium on User Interface Software and Technology. pp. 283-292. UIST '10, ACM (2010)

3. Biet, M., Casiez, G., Giraud, F., Lemaire-Semail, B.: Discrimination of virtual square gratings by dynamic touch on friction based tactile displays. In: 2008 Symposium on Haptic Interfaces for Virtual Environment and Teleoperator Systems. pp. 41-48 (March 2008)

4. Biet, M., Giraud, F., Lemaire-Semail, B.: Squeeze film effect for the design of an ultrasonic tactile plate. IEEE Transactions on Ultrasonics, Ferroelectrics, and Frequency Control 54(12), 2678-2688 (December 2007)

5. Buxton, W., Hill, R., Rowley, P.: Issues and techniques in touch sensitive tablet input. In: Proceedings of the 12th Annual Conference on Computer Graphics and Interactive Techniques. pp. 215-224. SIGGRAPH '85, ACM (1985)

6. Choi, S., Kuchenbecker, K.J.: Vibrotactile display: Perception, technology, and applications. Proceedings of the IEEE 101(9), 2093-2104 (September 2013)

7. Giraud, F., Amberg, M., Lemaire-Semail, B., casiez, G.: Design of a transparent tactile stimulator. In: 2012 IEEE Haptics Symposium (HAPTICS). pp. 485-489 (March 2012)

8. Goguey, A., Vogel, D., Chevalier, F., Pietrzak, T., Roussel, N., Casiez, G.: Leveraging finger identification to integrate multi-touch command selection and parameter manipulation. International Journal of Human-Computer Studies 99, 21 - 36 (2017)

9. Gueorguiev, D., Vezzoli, E., Sednaoui, T., Grisoni, L., Lemaire-Semail, B.: Feeling multiple edges: The tactile perception of short ultrasonic square reductions of the finger-surface friction. In: 2017 IEEE World Haptics Conference (WHC). pp. 125129 (June 2017)

10. Gueorguiev, D., Bochereau, S., Mouraux, A., Hayward, V., Thonnard, J.L.: Touch uses frictional cues to discriminate flat materials. Scientific Reports 6, 25553 (May 2016)

11. Gueorguiev, D., Vezzoli, E., Mouraux, A., Lemaire-Semail, B., Thonnard, J.L.: The tactile perception of transient changes in friction. Journal of The Royal Society Interface 14(137) (2017)

12. Hughes, B., Wang, J., Rosic, D., Palmer, K.: Texture gradients and perceptual constancy under haptic exploration. In: Second Joint EuroHaptics Conference and Symposium on Haptic Interfaces for Virtual Environment and Teleoperator Systems (WHC'07). pp. 66-71 (March 2007)

13. Kalantari, F., Berthaut, F., Grisoni, L.: Enriching Musical Interaction on Tactile Feedback Surfaces with Programmable Friction. In: International Symposium on Computer Music Multidisciplinary Research (CMMR 2017). Springer-Verlag, Porto, Portugal (2017) 
14. Kalantari, F., Grisoni, L., Giraud, F., Rekik, Y.: Finding the minimum perceivable size of a tactile element on an ultrasonic based haptic tablet. In: Proceedings of the 2016 ACM on Interactive Surfaces and Spaces. pp. 379-384. ISS '16, ACM (2016)

15. Kalantari, F., Lank, E., Rekik, Y., Grisoni, L., Giraud, F.: Determining the Haptic Feedback Position for Optimizing the Targeting Performance on Ultrasonic Tactile Displays. In: IEEE Haptics Symposium (HAPTICS 2018). San Fransisco, United States (2018)

16. Lederman, S.J., Klatzky, R.L.: Haptic perception: A tutorial. Attention, Perception, \& Psychophysics 71(7), 1439-1459 (October 2009)

17. Leek, M.R.: Adaptive procedures in psychophysical research. Perception \& Psychophysics (2001)

18. Linjama, J., MÃd'kinen, V.: E-sense screen: Novel haptic display with capacitive electrosensory interface. In: International Workshop on Haptic and Audio Interaction Design (HAID '09). Springer (2009)

19. Masson, D., Goguey, A., Malacria, S., Casiez, G.: Whichfingers: Identifying fingers on touch surfaces and keyboards using vibration sensors. In: Proceedings of the 30th Annual ACM Symposium on User Interface Software and Technology. pp. 41-48. UIST '17, ACM (2017)

20. Nefs, H.T., Kappers, A.M.L., Koenderink, J.J.: Amplitude and spatial-period discrimination in sinusoidal gratings by dynamic touch. Perception 30(10), 1263-1274 (2001)

21. Purves, D.: Neuroscience, 2nd Edition. Oxford University Press (2001)

22. Shultz, C.D., Peshkin, M.A., Colgate, J.E.: Surface haptics via electroadhesion: Expanding electrovibration with johnsen and rahbek. In: 2015 IEEE World Haptics Conference (WHC). pp. 57-62 (June 2015)

23. Skedung, L., Arvidsson, M., Young Chung, J., Stafford, C., Berglund, B., Rutland, M.: Feeling small: Exploring the tactile perception limits. Scientific reports 3, 2617 (2013)

24. Treutwein, B.: Adaptive psychophysical procedures. Vision Research 35(17), 2503 $-2522(1995)$

25. Verrillo, R.T., Fraioli, A.J., Smith, R.L.: Sensation magnitude of vibrotactile stimuli. Perception \& Psychophysics 6(6), 366-372 (November 1969)

26. Vezzoli, E., Sednaoui, T., Amberg, M., Giraud, F., Lemaire-Semail, B.: Texture Rendering Strategies with a High Fidelity - Capacitive Visual-Haptic Friction Control Device. In: Haptics: Perception, Devices, Control, and Applications, EuroHaptics '16. Springer (2016)

27. Wijekoon, D., Cecchinato, M.E., Hoggan, E., Linjama, J.: Electrostatic modulated friction as tactile feedback: Intensity perception. In: Proceedings of the EuroHaptics'12. pp. 613-624. Springer (2012)

28. Winfield, L., Glassmire, J., Colgate, J.E., Peshkin, M.: T-pad: Tactile pattern display through variable friction reduction. In: Second Joint EuroHaptics Conference and Symposium on Haptic Interfaces for Virtual Environment and Teleoperator Systems (WHC'07). pp. 421-426 (March 2007)

29. Yoshioka, T., BensmaÃáa, S.J., Craig, J.C., Hsiao, S.S.: Texture perception through direct and indirect touch: An analysis of perceptual space for tactile textures in two modes of exploration. Somatosensory \& Motor Research 24(1-2), 53-70 (2007) 